\title{
SURGICAL TREATMENT FOR PULMONARY METASTASES OF OSTEOSAR-COMA
}

doi: 10.2478/rojost-2018-0073

\author{
I.M. Rădulescu \\ Thoracic Surgery Department, University Emergency Hospital, Bucharest, Romania
}

Introduction. Osteosarcomas represent $40 \%$ of bone sarcomas and occur mainly in children and young adults. They are tumors with a high degree of pulmonary metastasis.

Pulmonary metastases occur in about $80 \%$ of the relapsed patients after primary tumor therapy, whether or not they have received chemotherapy. As therapeutic solutions, both first intention resection of the metastasis and the post-chemotherapy resection or repeated resections in each relapse, represent satisfactory results with prolongation of survival.

Material and method. The present study describes a group of 17 patients who were surgically treated for osteosarcoma with different localizations and who presented themselves in the thoracic surgery department during 2011-2016 with suspicion of pulmonary metastases. Three of them were at their second intervention for metastasis resection. Following investigations, 4 of them (23.5\%) could not benefit from surgical resection of pulmonary metastases (lesional extension with postoperative vital risk or technical impossibility of metastatic excision). Surgical interventions were curative; lobectomies were performed in 4 cases (30.7\%), atypical resections in 8 cases $(61.6 \%)$ and one pneumonectomy (7.7\%). Postoperative progression was favorable in all 13 patients.

Discussions and conclusions. Surgical excision of osteosarcoma pulmonary metastases can prolong survival and sometimes even cure disease. In order to benefit from the intervention, the patient should be served by a thoracic surgery clinic in the shortest possible time after the discovery of the pulmonary nodules. Thus, the need for thoracic imaging is required in patients treated for osteosarcoma. Late presentation makes the intervention impossible, particularly because of the lesional extension in the lung parenchyma or the invasion of the adjacent structures, extension given by the number of metastases or their size.

Keywords: pulmonary metastases, osteosarcoma, thoracic surgery 